\title{
Beanstandungen durch Kostenerstatter im ersten Halbjahr 2015
}

\begin{abstract}
Gab es Ende 2012 rund 239 substanzielle Beanstandungsthemen, die von Kostenerstattern als Anlass oder zur Begründung von Erstattungsminderungen herangezogen wurden, sind es im ersten Halbjahr 2015 nun 482 unterschiedliche Themen. Waren vor beziehungsweise zu Beginn der Anwendung der GOZ 12 die Themen "Bemessen/Begründen" (18 Prozent), "Analogien“ (zehn Prozent) und "Material- und Laborkosten" (acht Prozent) führend, so sind es 2015 zwar immer noch die Themen "Analogie" und "Materialund Laborkosten", aber ein neues Thema ist hinzugekommen.
\end{abstract}

\section{Skandal oder Tragödie?}

Die Beanstandungen rund um die Nr. 2197 GOZ (adhäsive Befestigung) in allen Varianten - gegen alle und jede Verbindung mit einer Grundleistung - haben extrem zugenommen und nehmen nun den dritten Platz in der Beanstandungsriege ein.

Wer zu verantworten hat, dass zunächst einmal bei den Nrn. $2060 \mathrm{ff}$. (Kompositrestaurationen) und dann immer öfter eine „Mehraufwandvergütung" als Bestandteil erst einer, dann zuletzt fast jeder Grundleistung bezeichnet werden kann, der hat Tür und Tor geöffnet für so waghalsige Kontra-Argumentation wie zum Beispiel:

„In der seit 1987 genau so beschriebenen und seither völlig unverändert bewerteten Leistung ,Eingliedern eines Klebebrackets' (6100 GOZ) ist die seit GOZ-Novellierung 2012 neue

\begin{tabular}{|l|c|c}
\hline Beanstand. 1.HIbj. 2014 & Anteil in \% & Platzierung \\
\hline Analogie & $14,4 \%$ & $\mathbf{1}$ \\
\hline Mat.-Laborkosten & $10,9 \%$ & $\mathbf{2}$ \\
\hline 2197 adhäsive Befestigung & $9,7 \%$ & $\mathbf{3}$ \\
\hline Bemessen/ Begründen & $8,5 \%$ & $\mathbf{4}$ \\
\hline Wurzelbehandlung & $7,8 \%$ & $\mathbf{5}$ \\
\hline 8000 ff. Indikation & $4,7 \%$ & $\mathbf{6}$ \\
\hline Verbrauchsmaterial & $4,5 \%$ & $\mathbf{7}$ \\
\hline Röntgeneinwände (z.B.dig.Rö) & $3,9 \%$ & $\mathbf{8}$ \\
\hline Schleimhautplastiken & $2,6 \%$ & $\mathbf{9}$ \\
\hline Beratungen nach GOÄ & $2,6 \%$ & $\mathbf{1 0}$ \\
\hline Formalfehler Rechnungen & $2,5 \%$ & 11 \\
\hline 2180 Aufbaufüllung etc. & $2,3 \%$ & 12 \\
\hline 5170 indiv. Löffel & $2,1 \%$ & 13 \\
\hline Provisorien 2270-7100 & $1,8 \%$ & 14 \\
\hline$\S 2$ (1,2) Vereinbarung & $1,5 \%$ & 15 \\
\hline O090, 0100 Anästhesien & $1,2 \%$ & 16 \\
\hline supraging. Belagentfernung & $1,1 \%$ & 17 \\
\hline Knochenreg./-management & $1,0 \%$ & 18 \\
\hline Unterlagenanforderung etc. & $0,8 \%$ & 19 \\
\hline Heil- und Kostenplan & $0,8 \%$ & 20 \\
\hline Implantatinsertion/ Abutment & $0,8 \%$ & 21 \\
\hline Untersuchungen Ä5, Ä6 etc. & $0,8 \%$ & 22 \\
\hline GOZ-/GOÄ-Zuschlag falsch & $0,7 \%$ & 23 \\
\hline Notwendigkeit & $0,6 \%$ & 24 \\
\hline 1040 PZR Prof. Zahnrein. & $0,6 \%$ & 25 \\
\hline
\end{tabular}

Tabelle der wichtigsten 25 Beanstandungsthemen (Zusammenfassungen) ,Mehraufwandvergütung' nach Nr. 2197 (bereits seit 25 Jahren) enthalten." - Der Nichterstatter verkürzt diese Aussage nach seiner Manier: „In der Leistung ,Klebebracket' ist adhäsive Befestigung enthalten" (ansonsten würde das Bracket nicht kleben). Dass das „Aufkleben“ des Jahres 1988 und die adhäsiv-infiltrative Befestigung in Form einer „Verbundklebung" dreidimensional unterschiedlich sind, müssten selbst Laien nachvollziehen können. Und eigentlich auch den Merksatz: In der Grundleistung kann logisch niemals Mehraufwandvergütung bereits enthalten sein.

Die Tendenz zur immer weiteren Aufsplittung der Beanstandungen in Einzelthemen scheint gestoppt, allerdings findet ein deutlicher Themenaustausch statt: Neue Themen kommen, alte sind auf einmal des Vorbringens nicht mehr wert. Es wird offenkundig seitens der Erstatter systematisch ausprobiert, wo wirksam zu sparen ist. Kostenerstatter beanstanden prozentual immer noch zirka doppelt so häufig, wie vor beziehungsweise zu Beginn der Geltung der GOZ 12 .

Die Tabelle (unten) beruht auf einer Zusammenfassung unter allgemeinen Überschriften. So verbergen sich hinter der Überschrift „Analogie“ zurzeit 59 Einzelthemen. Es sind führend völlig unbestimmte und unbegründete Einwände mit pauschaler Ablehnung der Analogberechnung oder sogar mit eigenmächtiger Festlegung einer „genehmen“, stets niedriger bewerteten Analogziffer. Dann kommen die Themen „Ausräumen, Fragment-/ Hindernisentfernung “ im Wurzelkanal, und es folgt der präendodontische Aufbau, dann der (ominöse) "geschichtete" SDAKompositaufbau als Analogleistung und die ,antimikrobielle photodynamische Therapie“ (aPDT, PDT, PT, PACT etc.). Einwände gegen „Kariesdetektoranwendung“ und ähnliche Kariesdiagnostik sind in der Häufigkeit zuletzt wieder gestiegen. Laseranwendung zieht immer mehr Widerspruch auf sich.

\section{Pauschalablehnungen nehmen stark zu}

Erschreckend ist die Zunahme von kaum oder total unbegründeten Pauschalablehnungen. Nur wenige Versicherungen tun sich auf diesem Feld hervor. Es ist zum Beispiel allseits bekannt, dass die Beihilfe die Spitzenposition bei der substanziell unbegründeten Ablehnung von höheren Gebührensätzen einnimmt - Bescheid: „Der Regelsatz wurde überschritten.“

Die Beihilfe ist auch führend bei Pauschalablehnung funktionsanalytischer/-therapeutischer Leistungen, aber die Post besetzt den Spitzenplatz bei der vollständig unbegründeten Ablehnung von Analogleistungen. Bei der wenig begründeten Ablehnung von Material- und Laborkosten tun sich derzeit besonders die HuK Coburg (pauschal), auch die Central-Krankenversicherung (detailliert), die DBV (Sachkostenliste), die Versicherungskammer Bayern beziehungsweise Bayrische Beamtenkrankenkasse (Liste der angemessenen Laborkosten), jetzt weniger die Allianz und kaum noch die Barmenia hervor.

(Es wird der ZA Zahnärztliche Abrechnungsgesellschaft AG für die Überlassung der statistischen Zahlen gedankt. Diese genau dokumentierten Zahlen sind trotz einer Basis von einigen hunderttausend Rechnungen nicht gesichert repräsentativ für das Gesamtgeschehen im Privatabrechnungsbereich in Deutschland. Es wäre von hohem Interesse, wenn weitere Institutionen vergleichbare Auswertungen vorlegen könnten.)

C) ZA 2015, P.E. 